\title{
A Novel Technique for Identifying Patients with ICU Needs Using Hemodynamic Features
}

\author{
A. Jalali, ${ }^{1}$ P. Ghorbanian, ${ }^{1}$ A. Ghaffari, ${ }^{2}$ and C. Nataraj ${ }^{1}$ \\ ${ }^{1}$ Department of Mechanical Engineering, Villanova University, 800 Lancaster Avenue, Villanova, PA 19085, USA \\ ${ }^{2}$ Department of Mechanical Engineering, K.N.Toosi University of Technology, No. 19, Pardis street, Mollasadra Avenue, \\ Vanak Square, Tehran 19991, Iran
}

Correspondence should be addressed to A. Jalali, ali.jalali@villanova.edu

Received 1 August 2011; Accepted 11 November 2011

Academic Editor: Hak-Keung Lam

Copyright (c) 2012 A. Jalali et al. This is an open access article distributed under the Creative Commons Attribution License, which permits unrestricted use, distribution, and reproduction in any medium, provided the original work is properly cited.

\begin{abstract}
Identification of patients requiring intensive care is a critical issue in clinical treatment. The objective of this study is to develop a novel methodology using hemodynamic features for distinguishing such patients requiring intensive care from a group of healthy subjects. In this study, based on the hemodynamic features, subjects are divided into three groups: healthy, risky and patient. For each of the healthy and patient subjects, the evaluated features are based on the analysis of existing differences between hemodynamic variables: Blood Pressure and Heart Rate. Further, four criteria from the hemodynamic variables are introduced: circle criterion, estimation error criterion, Poincare plot deviation, and autonomic response delay criterion. For each of these criteria, three fuzzy membership functions are defined to distinguish patients from healthy subjects. Furthermore, based on the evaluated criteria, a scoring method is developed. In this scoring method membership degree of each subject is evaluated for the three classifying groups. Then, for each subject, the cumulative sum of membership degree of all four criteria is calculated. Finally, a given subject is classified with the group which has the largest cumulative sum. In summary, the scoring method results in $86 \%$ sensitivity, $94.8 \%$ positive predictive accuracy and $82.2 \%$ total accuracy.
\end{abstract}

\section{Introduction}

Hemodynamic instability is most commonly associated with abnormal or unstable blood pressure (BP), especially hypotension, or more broadly associated with inadequate global or regional perfusion. Inadequate perfusion may compromise important organs, such as the heart and the brain, due to limits on coronary and cerebral autoregulation, and may cause life-threatening illnesses or even death. Therefore, it is crucial to identify patients who are likely to become hemodynamically unstable for early detection and treatment of these life-threatening conditions [1]. The modern intensive care units (ICUs) typically employ continuous hemodynamic monitoring (e.g., heart rate (HR) and invasive arterial BP measurements) to track the state of patients. However, clinicians in a busy ICU are overwhelmed with the task of assimilating and interpreting the tremendous volumes of data into working hypotheses. Consequently, it is important to have automated algorithms that can accurately process and classify the large amount of monitoring data and identify patients who are on the verge of becoming unstable [1].

Modern ICUs are equipped with a large array of alarmed monitors and devices which are used in an attempt to detect clinical changes at the earliest possible moment, so as to prevent any further deterioration in a patient's condition. The effectiveness of these systems depends on the sensitivity and specificity of the alarms as well as on the responses of the ICU staff to the alarms. However, when large numbers of alarms are either technically false, or true, but clinically irrelevant, response efficiency can be decreased, reducing the quality of patient care and increased patient (and family) anxiety [2].

Medical and technical progress has extended the therapeutic possibilities of ICUs tremendously. A multitude of devices are available for monitoring and treatment in an individual assembly according to the requirements of the situation [3]. Due to limited physiological monitoring and a 
patient's individual pathophysiology, intensive care medicine has to cope with a high amount of uncertainty. Unusual circumstances caused by patients, clinicians, and technology occur frequently and must be controlled and managed adequately to prevent a bad outcome and to achieve system reliability [3].

Cao et al. [1] have used ICU minute-by-minute heart rate (HR) and invasive arterial blood pressure (BP) monitoring trend data collected from the MIMIC II database to predict hemodynamic instability at least two hours before a major clinical intervention. They derived additional physiological parameters of shock index, rate pressure product, heart rate variability, and two measures of trending based on HR and BP, and they applied multivariable logistic regression modeling to carry out classification and implemented validation via bootstrapping, resulting in $75 \%$ sensitivity and $80 \%$ specificity. Eshelman et al. [4] have developed an algorithm for identifying ICU patients who are likely to become hemodynamically unstable. Their algorithm consists of a set of rules that trigger alerts and uses data from multiple sources; it is often able to identify unstable patients earlier and with more accuracy than alerts based on a single threshold. The rules were generated using the machine learning techniques of support vector machines and neural network and were tested on retrospective data in the MIMIC II ICU database, yielding a specificity of approximately $90 \%$ and a sensitivity of $60 \%$.

Several investigations have been reported in the literature in the area of cardiovascular fault diagnosis using hemodynamic features. Javorka et al. [18] compared heart rate and blood pressure variability among young patients with type I diabetes mellitus (DM) and control subjects by using Poincare plots, which are the standard tools of nonlinear dynamic analysis. They found significant reduction of all HRV Poincare plot measure in patients with type I diabetes mellitus, indicating heart rate dysregulation. The study carried out by Pagani et al. [5] concerned patients suffering from hypertension. They showed that baroreflex gain decreases with the presence of hypertension. Blasi et al. [6] studied the effects of arousal from sleep on cardiovascular variability. They performed time-varying spectral analyses of heart rate variability (HRV) and blood pressure variability (BPV) records during acoustically induced arousals from sleep. They found that arousal-induced changes in parasympathetic activity are strongly coupled to respiratory patterns, and that the sympathoexcitatory cardiovascular effects of arousal are relatively long lasting and may accumulate if repetitive arousals occur in close succession.

Advances in knowledge-based systems have also enhanced the functionality of intelligent alarm systems and ICU needed patient detection. Using the knowledge of a domain expert to formulate rules or an expertly classified data set to train an adaptive algorithm has proven useful for intelligent processing of clinical alarms [2]. Expert systems such as neural network [7], knowledge-based decision trees $[8,9]$, and neurofuzzy systems [10] that encode the decisions of an expert clinician all show significant statistical improvement in the classification of alarms and ICU needed patients. Singh and Guttag [11] proposed a classification algorithm based on a decision tree method for cardiovascular risk stratification. They have shown that the decision tree method can improve the performance of the classification algorithm. They have reported that the decision tree models outperform the radial basis function (RBF) kernel-based support vector machine (SVM) classifiers. Timms et al. [12] have used a Mock circulation loop for hemodynamic modeling of the cardiovascular system in order to test cardiovascular devices, which are used in the ICU and can provide a better indication of patient's condition for the nursing staff. Also, Laramee et al. [2] have described an integrated systems methodology to extract clinically relevant information from physiological data. Such a method would aid significantly in the reduction of false alarms and provide the nursing staff with a more reliable indicator of patient condition.

Regarding suitable classifier structures, several studies have been reported in the literature. Ghorbanian et al. [13] proposed an algorithm based on a neural network classifier for heart arrhythmias detection. Their results show that the multilayer perceptron neural network (MLPNN) structure is a strong and precise classifier. They used several preprocessing techniques in their algorithm to improve the performance of the NN classifier. Rajendra Acharya et al. [14] proposed an algorithm based on a neural network classifier and fuzzy cluster for classification of heart arrhythmias. They compared these two classifiers and they reported that the fuzzy cluster is a better classifier in comparison with the neural one. Also, Özbay et al. [15] proposed a comparative study of the classification accuracy cardiovascular diseases using a well-known neural network architecture, a multilayered perceptron (MLP) structure, and a new fuzzy clustering NN architecture (FCNN) for early diagnosis. Based on their test results, they suggested that a new proposed FCNN architecture can generalize better than ordinary MLP architecture and also learn better and faster.

The method for classification of subjects into two categories of normal and abnormal subjects, as described in this paper, is based on the hypothesis that there should be differences between the hemodynamic data collected from normal subjects and abnormal patients. This hypothesis is constructed on the same foundation as all developed scoring methods for ICU patients. The idea behind all patient scoring methods in ICU is that critically ill patients in ICU are typically characterized by disturbance of the body's homeostasis. These disturbances can be estimated by measuring to what extent one or many physiologic variables differ from the normal range [16].

\section{Methodology}

While this paper shares some fundamental ideas with traditional scoring methods, the proposed method differs from them in two key areas. The first difference comes from fact that the patient scoring methods are based on the wide variety of data ranging from cardiovascular and respiratory systems to neurologic and renal systems variables. However, in our method we use a small subset of hemodynamic data, namely, HR and SBP. The principal objection to this could be that such a small amount of data could be insufficient 
for identifying the patient state; the answer to this objection leads us to the second major difference of the proposed method with the scoring methods. Scoring methods just look at the data as they are being collected in the ICU; they ignore information hidden in the different time scales. In our proposed method on the other hand, this hidden information is extracted which gives us better insight into the patient's physiological condition.

The data used in this study is collected from the Physionet database. Data are collected from two databases: MIT-BIH Polysmonographic and MIMIC II databases within Physionet archive. Twenty-five subjects from these databases were collected for training. For each subject, ECG signal and blood pressure waveform, in a five-hour range of the total data were collected. For the first part of the study, the HR and SBP series for each subject are derived from ECG and arterial pressure waveforms, respectively.

The algorithm of the method developed in this study is shown in Figure 1. The proposed algorithm consists of two stages: training and testing stages. In the training stage, 25 subjects' data are used for the training purpose of the algorithm. In this stage and after collecting the data, four features which highlight the differences between normal subjects and patients, are extracted from data. We then define four criteria based on the extracted features. These four criteria which form the basis of our classification algorithm are: circle criterion, estimation error criterion, Poincare care plot deviation, and autonomic response delay criterion. In the next step and for the task of classification, we define three groups, namely, healthy, high risk, and patient. Then we design three fuzzy membership functions for each criterion to find the subject degree of membership to each group. Finally, a scoring method is developed based on the degree of membership of each case, and subjects are classified based on this scoring method.

In the following sections, we provide a step-by-step description of our method, beginning with the definition of the proposed criteria.

2.1. Circle Criterion. To evaluate the differences between healthy and patients, the SBP against HR diagram for each subject is plotted. Figure 2 shows these plots for healthy and patient cases. Clearly, the plots show a significant difference between normal subjects and abnormal patients: the data for normal subjects are concentrated, while those of the patients are scattered.

The mean value of SBP and HR for each normal subject and abnormal patient is then calculated and plotted in one diagram. Figure 3 shows the mean values for all the subjects in one diagram. The principal difference between the two groups is quite clear. This diagram reveals the fact that there are differences between the HR and SBP data in normal subjects and abnormal ones. The plot shows that the data for the normal subjects is clustered and limited in a specific area, while those of the patients are spread out through the whole plot. The first criterion is named the "circle criterion". The center of the circle is located at point "O" where its coordinates are the mean values of HR and SBP of normal patients and, in this case, is $(83,120)$. The radius of this circle

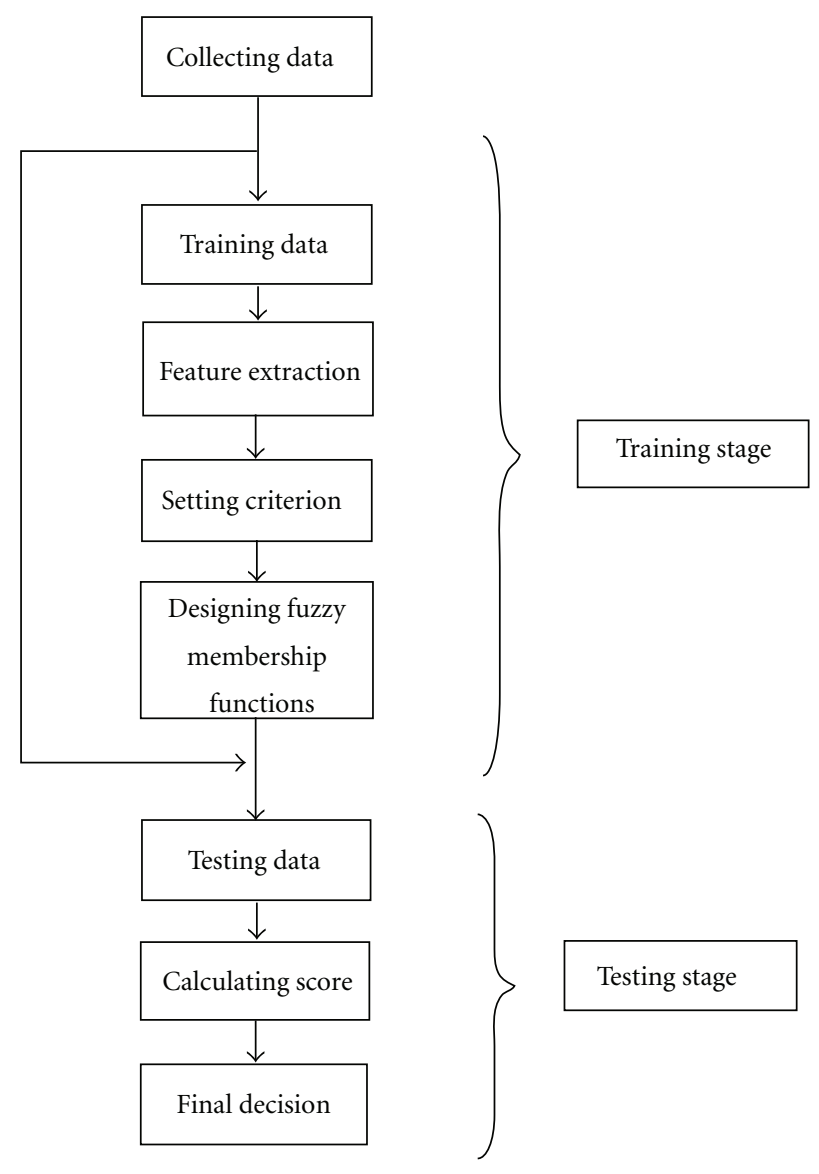

FIGURE 1: Schematic of the proposed algorithm. The proposed algorithm consists of two stages: training and testing. In the training stage 25 subjects' data are used to extract features to classify patients from healthy subjects. In the test stage, subjects will be divided into three predefined groups of healthy, high risk, and patient, based on their assigned score.

is calculated based on Euclidian distance between the center and the outer limit of the circle.

A given subject would be considered to be a patient if its corresponding mean (HR and SBP) point is out of the healthy subject's circle (the limited area).

2.2. Estimation Error Criterion. As the second feature, a system identification method is used for the prediction of the next HR based on the current and previous HR and SBP data. A nonlinear ARX or NARX model is employed to estimate HR series [17]. NARX models in general are represented by the following equation:

$$
\begin{gathered}
y(t)=F\left(y(t-1), y(t-2), \ldots, y\left(t-n_{a}\right), u\left(t-n_{k}\right), \ldots,\right. \\
\left.u\left(t-n_{k}-n_{b}+1\right)\right),
\end{gathered}
$$

where, $y(t)$ and $u(t)$ are the output and input of the system, respectively. In (1) the matrix [ $\left[\begin{array}{lll}n_{a} & n_{b} & n_{k}\end{array}\right]$ is the same as the order of the model. Model order is selected by use of the AInformation Criterion (AIC) method. This is the traditional 


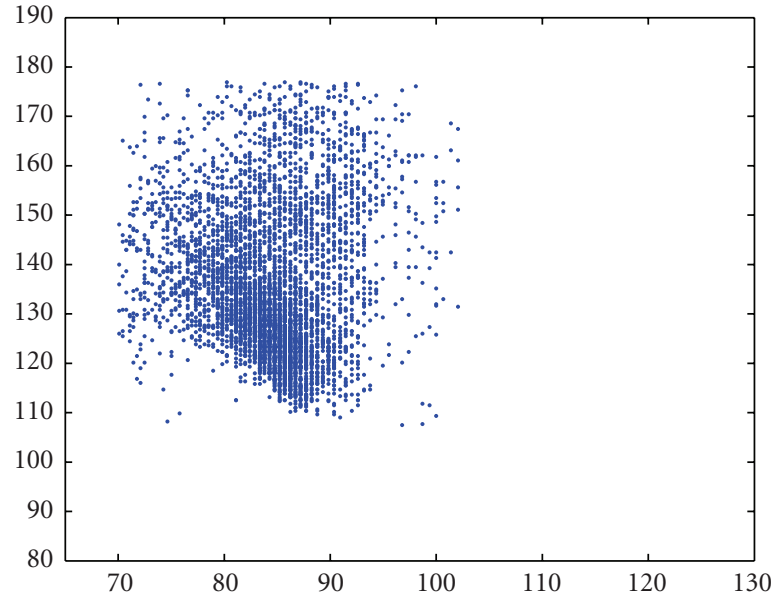

(a)

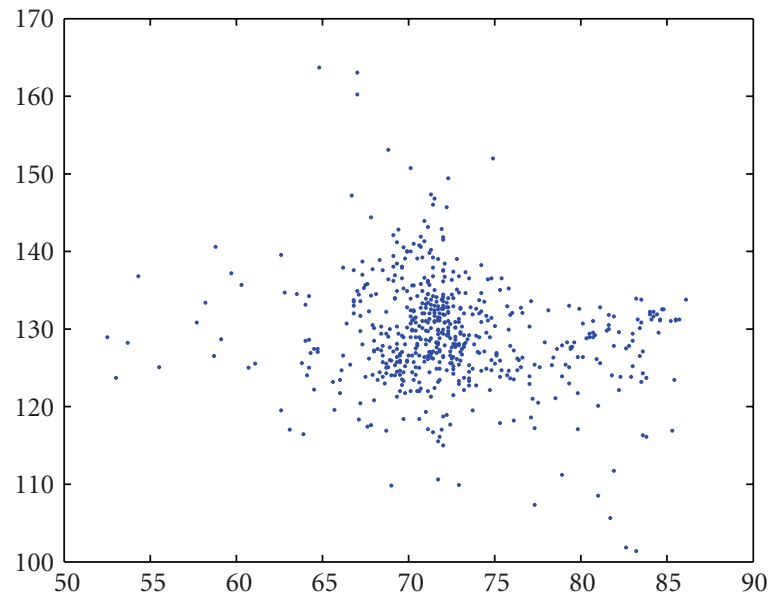

(b)

FIGURE 2: SBP against HR for a healthy (a) and an abnormal (b) case.

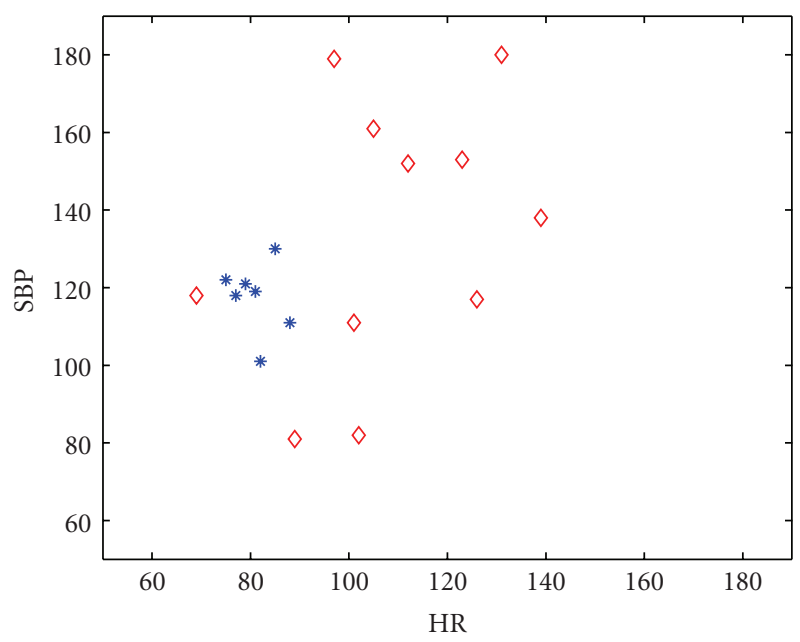

Figure 3: Mean values of SBP versus HR for all subjects.

method for model order selection in cardiovascular system identification research. Model order for data in this research has been calculated to be $\left[\begin{array}{lll}9 & 6 & 3\end{array}\right]$.

In this criterion, an artificial neuro fuzzy inference system (ANFIS) structure is employed for the identification purpose. The model has 15 inputs and one output. Membership functions for inputs are designed based on physiological facts. Since the nervous system consists of sympathetic and parasympathetic nerves, for each input, two generalized bellshaped membership functions are assigned to designate the sympathetic and parasympathetic functions.

The system identification results are described in Table 1. The results in this table show that differences exist in the normalized root mean square error (NRMSE) with respect to the estimation of the HR for the two groups under study. In particular, the results indicate that NRMSE is smaller for normal subjects than for patients. These differences are due to the fact that the model is designed for normal subjects,
TABLE 1: Error estimation for identification of HR baroreflex.

\begin{tabular}{lccc}
\hline Group & Mean & Max & Min \\
\hline Normal & 0.193 & 0.238 & 0.119 \\
Abnormal & 0.367 & 0.473 & 0.263 \\
\hline
\end{tabular}

TABle 2: Deviation from line $y=x$ in Poincare plot.

\begin{tabular}{lcc}
\hline Group & Mean & Max \\
\hline Healthy & $8 \%$ & $13 \%$ \\
Patient & $19 \%$ & $24 \%$ \\
\hline
\end{tabular}

thus, the output of the model for patients has higher errors than that for normal subjects.

Based on these results and noting that the maximum error for healthy subject is 0.238 , while the minimum error for patient is 0.263 , we define a second criterion called "estimation error criterion." According to this criterion, the subject would be flagged as abnormal if the calculated error in HR estimation raise is more than 0.25 .

2.3. Poincare Plot Deviation. A Poincare plot, named after Henri Poincare, is used to quantify self-similarity in processes which are usually characterized by periodic functions. This plot is commonly used in heart rate variability (HRV) analysis. The Poincare plot is a graph in which each heart rate episode is plotted as a function of previous $\mathrm{HR}$, and then the line $y=x$ is fitted to the data. In [18], this method is also applied to classify patients with type I DM from healthy subjects. Drawing the Poincare plot for healthy and abnormal subjects, it is found that the deviation from the mentioned line is less in healthy subjects than in abnormal subjects. These plots are shown in Figure 4.

The deviation from the line $y=x$ in the Poincare plot for the two groups under study is shown in Table 2 . Therefore, we define the third criterion using this deviation to characterize abnormality. Based on this criterion, subjects 


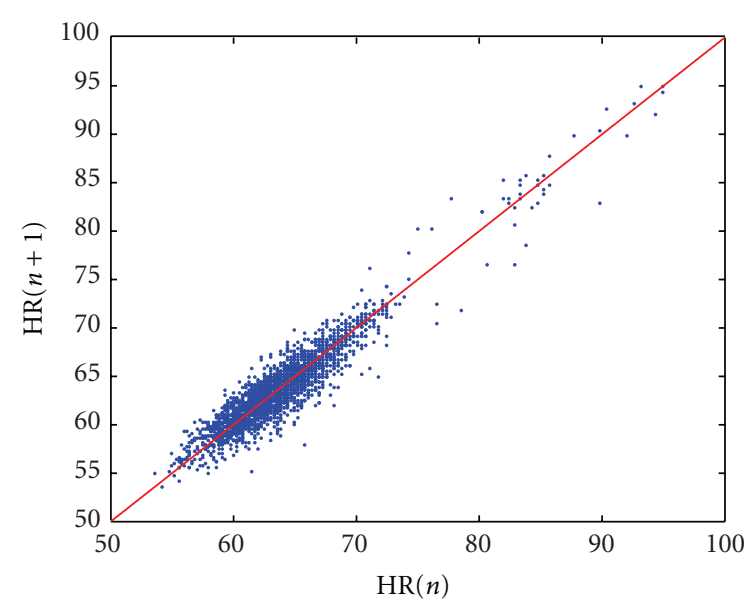

(a)

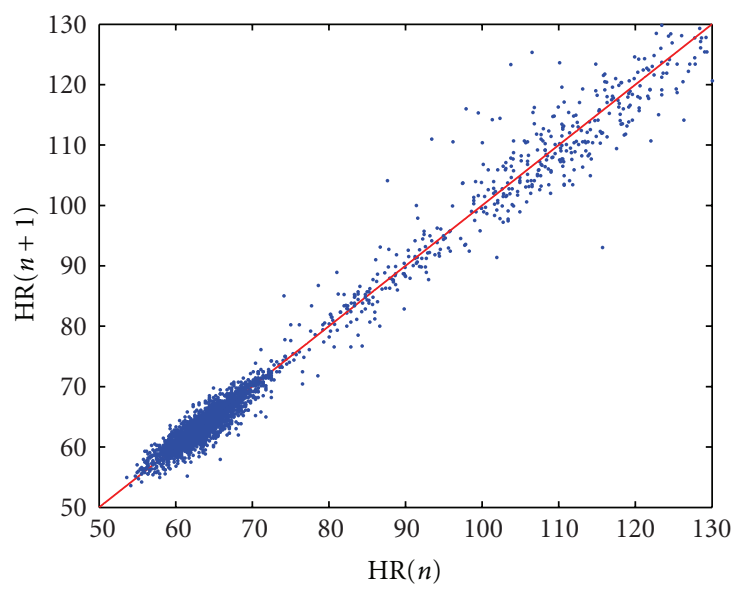

(c)

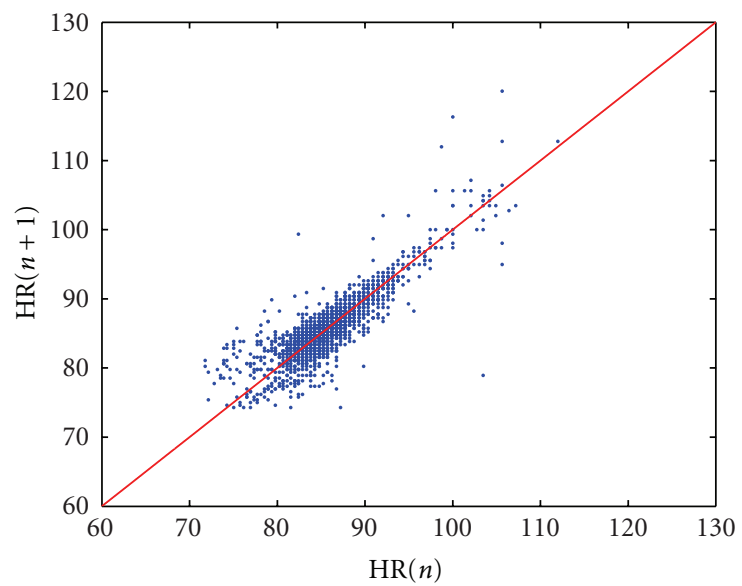

(b)

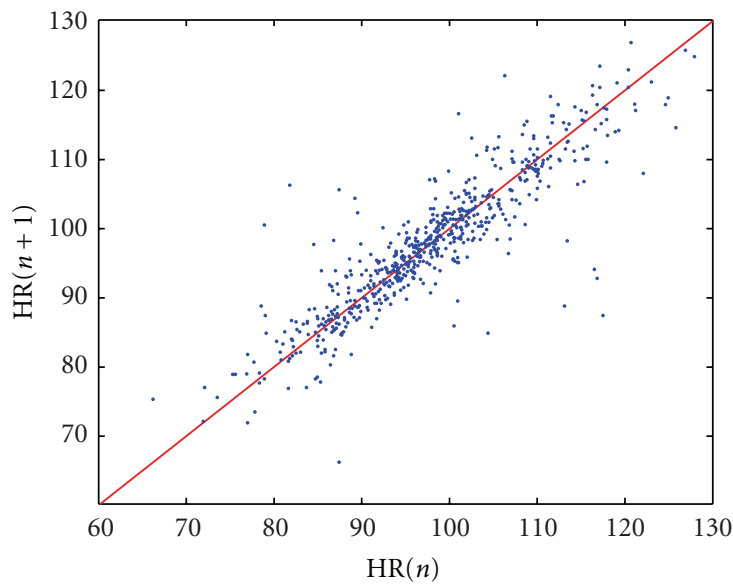

(d)

FIgURE 4: Poincare plots of HR for two healthy (up) and two abnormal (down) cases. The Poincare plot is plot of HR $(n+1)$ versus HR( $n$ ). Line $y=x$ is illustrated in all pictures.

would be called abnormal if deviation from line $y=x$ is more than $15 \%$.

2.4. Autonomic Response Delay Criterion. The normally occurring delay in the autonomic response to a stimulus has its origins in the parasympathetic nervous system. Calculating the delay for healthy subjects and patients, we can infer that response delays in abnormal subjects are remarkably higher than in healthy subjects. The results of calculating the delay in the autonomic response are shown in Figure 5. Fifteen abnormal patients and ten healthy subjects were involved in the training group.

The results of the delay calculations in the autonomic response are also presented in Table 3. Based on the above results, we define the fourth criterion where the subject is characterized as abnormal if the calculated delay in the autonomic response increases to more than 0.021 second.

After deriving the four criteria discussed above, an algorithm is designed to classify healthy subjects from patients. In the following section, we describe the proposed algorithm.
TABLE 3: Delay in autonomic response for two groups.

\begin{tabular}{lcc}
\hline Group & Mean delay $(\mathrm{sec})$ & Max delay $(\mathrm{sec})$ \\
\hline Healthy & 0.015 & 0.02 \\
Patient & 0.038 & 0.06 \\
\hline
\end{tabular}

\section{Scoring Method and Classification Algorithm}

Based on the evaluated criteria from training data, an algorithm is developed to automatically distinguish patients from healthy subjects. The algorithm is based on a fuzzy decisionmaking method. First, for each criterion, three Gaussian bell membership functions are designed as an indicator of three major groups: healthy, high risk, and patient. Since this algorithm is designed for clinical use and since there exists a high degree of uncertainty in clinical applications, we added the high-risk groups to our predefined healthy and patient groups, to account the cases that do not completely belong to the healthy or patient groups. For the training part, we first made a general guess for the shape of the membership functions. The membership functions during the training 
TABLE 4: Results of algorithm testing on the first group. The CS is abbreviation for cumulative sum.

\begin{tabular}{|c|c|c|c|c|c|}
\hline Case Number & Status & Healthy CS & High-risk CS & Patient CS & Category \\
\hline 1 & Patient & 0.4 & 0.7 & 2.8 & Patient \\
\hline 2 & Patient & 0.2 & 0.6 & 3.1 & Patient \\
\hline 3 & Healthy & 3.2 & 0.9 & 0.1 & Healthy \\
\hline 4 & Healthy & 2.5 & 1.4 & 0.2 & Healthy \\
\hline 5 & Patient & 0.1 & 1.1 & 2.6 & Patient \\
\hline 6 & Patient & 0.3 & 0.4 & 3.4 & Patient \\
\hline 7 & Patient & 0.3 & 2.2 & 1.6 & High risk \\
\hline 8 & Patient & 0.5 & 0.8 & 2.9 & Patient \\
\hline 9 & Healthy & 3.6 & 0.4 & 0.1 & Healthy \\
\hline 10 & Patient & 0.2 & 2.2 & 1.7 & High risk \\
\hline 11 & Patient & 0.2 & 0.6 & 3.0 & Patient \\
\hline 12 & Healthy & 2.7 & 0.9 & 0.3 & Healthy \\
\hline 13 & Patient & 0.3 & 0.6 & 3.2 & Patient \\
\hline 14 & Patient & 0.1 & 0.3 & 3.5 & Patient \\
\hline 15 & Patient & 2.9 & 0.7 & 0.3 & Healthy \\
\hline
\end{tabular}

TABLE 5: Results of algorithm testing on the second group.

\begin{tabular}{|c|c|c|c|c|c|}
\hline Case Number & Status & Healthy CS & High-risk CS & Patient CS & Category \\
\hline 1 & Healthy & 2.7 & 0.6 & 0.6 & Healthy \\
\hline 2 & Patient & 0.3 & 0.8 & 2.9 & Patient \\
\hline 3 & Patient & 0.2 & 0.8 & 3.0 & Patient \\
\hline 4 & Patient & 0.3 & 0.4 & 3.3 & Patient \\
\hline 5 & Healthy & 2.8 & 0.9 & 0.4 & Healthy \\
\hline 6 & Patient & 0.3 & 0.4 & 3.4 & Patient \\
\hline 7 & Patient & 0.4 & 0.8 & 2.8 & Patient \\
\hline 8 & Healthy & 1.5 & 1.9 & 0.6 & High risk \\
\hline 9 & Patient & 0.5 & 0.8 & 2.8 & Patient \\
\hline 10 & Patient & 0.4 & 0.6 & 3.1 & Patient \\
\hline 11 & Patient & 0.1 & 0.9 & 3.1 & Patient \\
\hline 12 & Patient & 0.2 & 2.5 & 1.2 & High risk \\
\hline 13 & Patient & 0.1 & 0.9 & 3.1 & Patient \\
\hline 14 & Patient & 0.4 & 0.5 & 3.1 & Patient \\
\hline 15 & Healthy & 3.1 & 0.8 & 0.2 & Healthy \\
\hline
\end{tabular}

TABLE 6: Results of algorithm testing on the third group.

\begin{tabular}{|c|c|c|c|c|c|}
\hline Case Number & Status & Healthy CS & High-risk CS & Patient CS & Category \\
\hline 1 & Patient & 0.1 & 0.7 & 3.2 & Patient \\
\hline 2 & Patient & 0.3 & 0.8 & 2.9 & Patient \\
\hline 3 & Healthy & 1.3 & 2.4 & 0.3 & High risk \\
\hline 4 & Patient & 0.2 & 0.7 & 3.1 & Patient \\
\hline 5 & Patient & 0.4 & 1.9 & 1.6 & High risk \\
\hline 6 & Patient & 0.4 & 0.8 & 2.9 & Patient \\
\hline 7 & Healthy & 2.7 & 0.9 & 0.3 & Healthy \\
\hline 8 & Patient & 0.1 & 0.4 & 3.5 & Patient \\
\hline 9 & Patient & 0.3 & 2.2 & 1.6 & High risk \\
\hline 10 & Patient & 0.4 & 0.6 & 3.1 & Patient \\
\hline 11 & Healthy & 3.0 & 0.7 & 0.3 & Healthy \\
\hline 12 & Patient & 0.4 & 0.8 & 2.7 & Patient \\
\hline 13 & Patient & 0.7 & 1.0 & 2.8 & Patient \\
\hline 14 & Healthy & 3.2 & 0.6 & 0.2 & Healthy \\
\hline 15 & Patient & 0.5 & 0.8 & 2.8 & Patient \\
\hline
\end{tabular}


TABLe 7: Results of testing the algorithm on Physionet database.

\begin{tabular}{lcccc}
\hline Group & I & II & III & All \\
\hline All & 15 & 15 & 15 & 45 \\
TP & 12 & 13 & 12 & 37 \\
FN & 3 & 1 & 2 & 6 \\
FP & 0 & 1 & 1 & 2 \\
Se $(\%)$ & 80 & 92.8 & 85.7 & 86 \\
PPA $(\%)$ & 100 & 92.8 & 92.3 & 94.8 \\
TA $(\%)$ & 80 & 86 & 80 & 82.2 \\
\hline
\end{tabular}

round then adapt their shape parameters to the incoming data for best classification performance. Now, the classifier is designed and ready for the testing stage. Figure 6 represents the adapted membership functions for each criterion based on the training data.

To test the developed algorithm, in the first step for each subject, all the mentioned features that form the basis of four criteria are extracted and used as an input for the four abnormality criteria. Then, for each criterion, the subject's degree of membership to all groups is evaluated. In this step, for each subject, we have 12 degrees of membership to the designed three groups, meaning four degrees of membership for each group. After evaluating the degree of memberships, the cumulative sum of the four degree of memberships of each group is calculated. In this stage, we have three numbers indicating the subject's degree of membership to each group. We call these numbers the subject's "score" for each group. A given subject will belong to the group whose score is the largest.

\section{Results}

From a total of seventy subject data which were collected from MIMIC II database, the algorithm was first trained with twenty-five subjects including ten healthy and fifteen patients. The training data was selected randomly to avoid bias towards a specific disease. Then, three groups of subjects were tested with each group consists of four healthy individuals and eleven patients.

The results of the test are presented in Tables 4, 5, and 6 . The proposed method was applied to 45 cases from Physionet database, containing 12 healthy subjects and 33 patients. From all cases, 37 cases were accurately detected, while there was one false detection. Furthermore, in five cases a patient subject was classified as high risk and, in two cases, a healthy subject was classified as high risk.

To assess the accuracy of the classifier, sensitivity (Se), positive predictive accuracy (PPA), and total accuracy (TA) have been calculated. These are defined as follows.

$$
\begin{gathered}
\mathrm{Se}=\frac{\mathrm{TP}}{(\mathrm{TP}+\mathrm{FN})}, \\
\mathrm{PPA}=\frac{\mathrm{TP}}{(\mathrm{TP}+\mathrm{FP})}, \\
\mathrm{TA}=\frac{\mathrm{TP}}{(\mathrm{TP}+\mathrm{FN}+\mathrm{FP})} .
\end{gathered}
$$

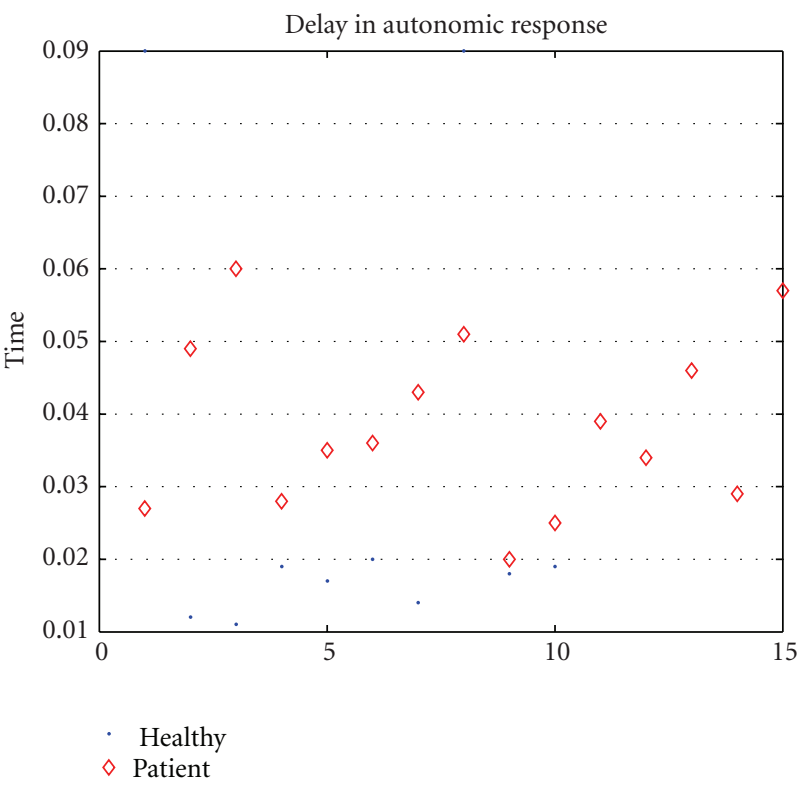

FIgURe 5: Delay in autonomic response.

Here, TP is the number of true positive detections, FN stands for the number of false negative detections, and FP stands for the number of false positive misdetections. Table 7 shows the overall result of the classification for all 45 cases of the 3 groups. The FP is the healthy subject who is misclassified as a high-risk subject, and FN is the patient who is misclassified as a high-risk subject. According to this table, the scoring method of the proposed algorithm results in $86 \%$ sensitivity, $94.8 \%$ positive predictive accuracy, and $82.2 \%$ total accuracy.

A comprehensive comparison between the results of different studies in the field of identified ICU needed patients by the use of hemodynamic features is very difficult since the database, signals under study, the algorithm structure, and the data processing methods are not the same in the various studies. However, in order to present an estimate of the performance of our algorithm and our classifier we show the results of this study versus the reported results of two other well-known studies in the area of ICU needed patients identifying in Table 8. As seen from this table, the algorithm in the present study shows reasonably accurate results and compares favorably with other studies. The goal of this study, which was identifying patients with ICU needs by use of the hemodynamic features, has clearly been achieved.

\section{Discussion and Conclusions}

In this paper a scoring method based on fuzzy logic and feature extraction is proposed to distinguish patients from healthy subjects. The method is based on the same principle that the ICU scoring methods follow: that of finding differences between hemodynamic data of healthy subjects and patients. Four different criteria are proposed to detect and identify patients from a group of subjects. For each criterion a fuzzy classifier is designed such that 


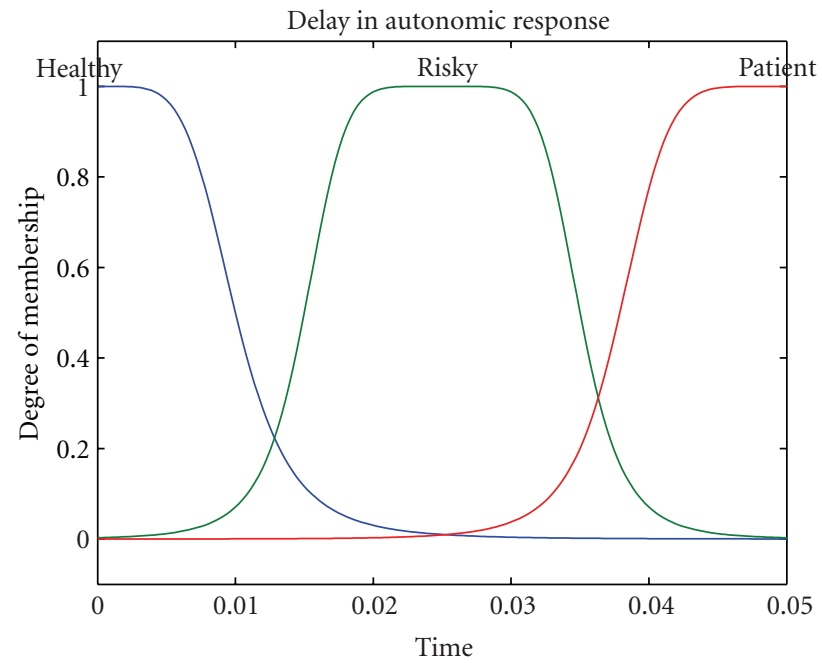

(a)

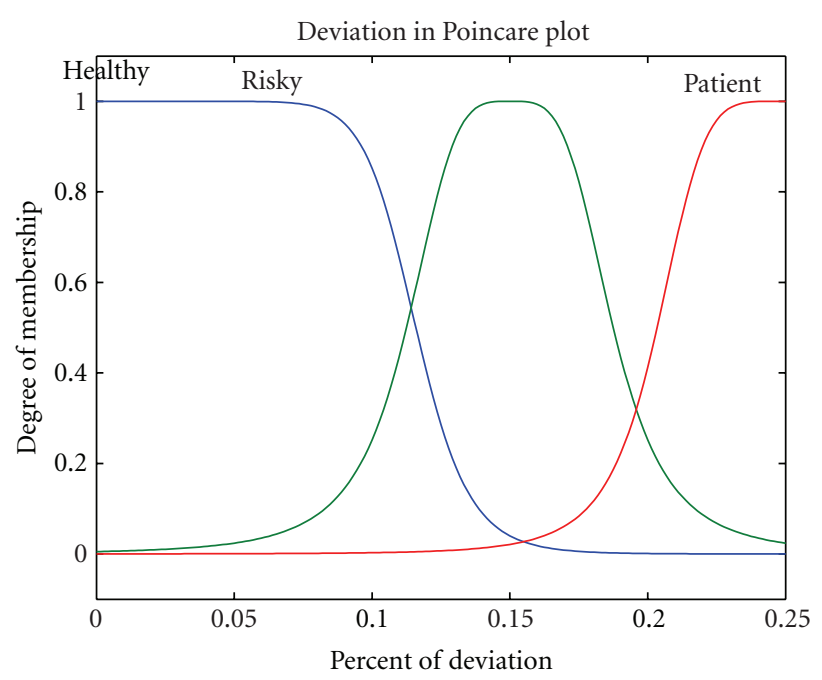

(c)

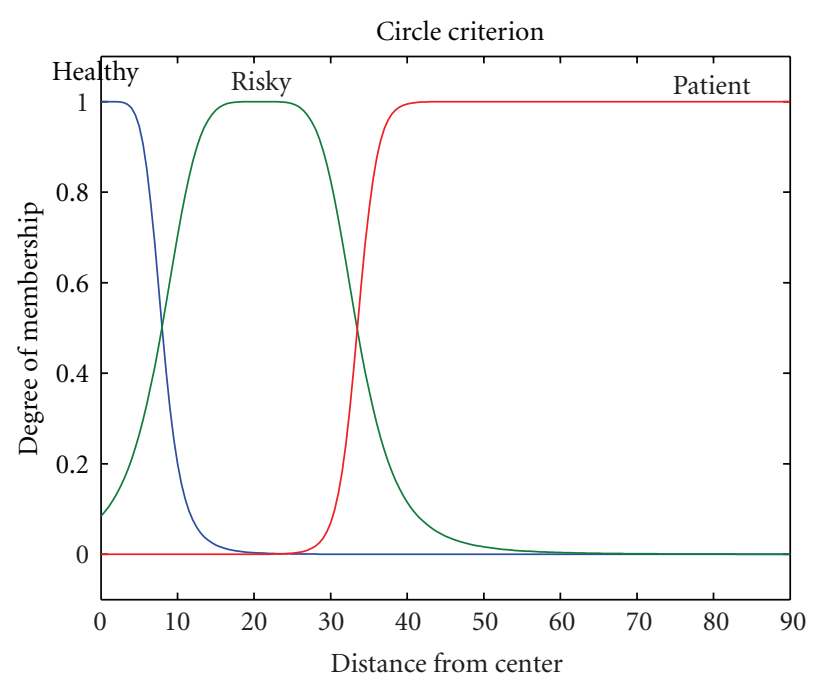

(b)

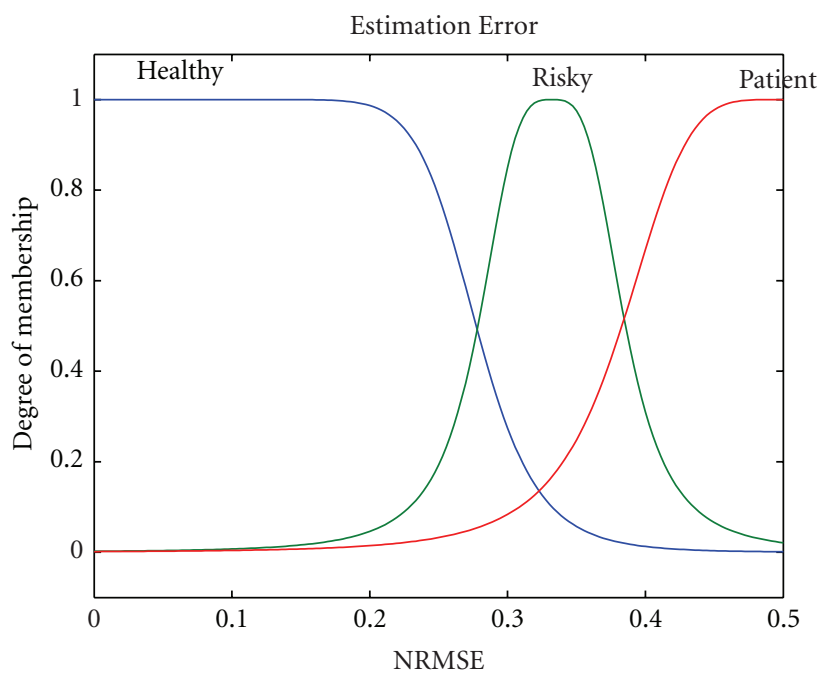

(d)

FIgURE 6: The designed membership functions for each criterion.

TABLE 8: Comparison of several classifier performances on MIMIC II ICU database (blank boxes have not been reported).

\begin{tabular}{lc}
\hline Study & Se (\%) \\
\hline Cao et al. [1] & 75 \\
Eshelman et al. [4] & 60 \\
This study & 86 \\
\hline
\end{tabular}

the individuals are classified into the healthy, high risk, and patient fuzzy groups. In other words, a given person may have a membership grade in all three classes. A score is assigned to the subject for a group as being equal to the sum of degree of memberships. The algorithm calculates a combined criterion based on the results of the four criteria to arrive at a classification decision for each individual.

It is shown that the algorithm is highly reliable and has been able to detect correctly all members of the first group.
It is also able to detect all eleven patients in each of the next two groups correctly. Only one of the healthy members in the second and third was classified as high-risk.

In this study, four different criteria were proposed and used in the proposed algorithm in order to detect the abnormalities in testing subjects. From each testing subject, various features were extracted and used as input for the criteria, and based on the results of all four criteria, a decision was made about the type of subject, as to whether he/she is normal, a high risk subject or a patient.

The proposed algorithm gave reliable results in detecting the ICU needed patients, but still needs to be improved. The difference between the proposed method in this study and other similar research in this field of study is that by using the presented algorithm in this study, existence of any abnormality in a patient will be found, while in most of similar studies in this area, a specific abnormality is found in a patient or among a database of subjects. Therefore, our 
results are more general and more useful from the point of view of clinical applications.

This method tends to be more detective rather than predictive, and this could be one drawback of the algorithm. Further investigations need to be carried out to improve its predicting ability.

\section{References}

[1] H. Cao, L. Eshelman, N. Chbat, L. Nielsen, B. Gross, and M. Saeed, "Predicting ICU hemodynamic instability using continuous multiparameter trends," in Proceedings of the 30th Annual International Conference of the IEEE Engineering in Medicine and Biology Society (EMBS '08), pp. 3803-3806, August 2008.

[2] C. B. Laramee, L. Lesperance, D. Gause, and K. McLeod, "Intelligent alarm processing into clinical knowledge," Proceedings of the Annual International Conference of the IEEE Engineering in Medicine and Biology Society, pp. 6657-6659, 2006.

[3] W. Friesdorf, B. Buss, and M. Gobel, "Monitoring alarmsthe key to patient's safety in the ICU?" Intensive Care Medicine, vol. 25, no. 12, pp. 1350-1352, 1999.

[4] L. J. Eshelman, K. P. Lee, J. J. Frassica, W. Zong, L. Nielsen, and M. Saeed, "Development and evaluation of predictive alerts for hemodynamic instability in ICU patients," Proceedings of the Annual Symposium proceedings/AMIA Symposium, pp. 379-383, 2008.

[5] M. Pagani, V. Somers, R. Furlan et al., "Changes in autonomic regulation induced by physical training in mild hypertension," Hypertension, vol. 12, no. 6, pp. 600-610, 1988.

[6] A. Blasi, J. Jo, E. Valladares, B. J. Morgan, J. B. Skatrud, and M. C. K. Khoo, "Cardiovascular variability after arousal from sleep: time-varying spectral analysis," Journal of Applied Physiology, vol. 95, no. 4, pp. 1394-1404, 2003.

[7] D. R. Westenskow, J. A. Orr, F. H. Simon, H. J. Bender, and H. Frankenberger, "Intelligent alarms reduce anesthesiologist's response time to critical faults," Anesthesiology, vol. 77, no. 6, pp. 1074-1079, 1992.

[8] C. L. Tsien, I. S. Kohane, and N. McIntosh, "Multiple signal integration by decision tree induction to detect artifacts in the neonatal intensive care unit," Artificial Intelligence in Medicine, vol. 19, no. 3, pp. 189-202, 2000.

[9] B. Müller, A. Hasman, and J. A. Blom, "Evaluation of automatically learned intelligent alarm systems," Computer Methods and Programs in Biomedicine, vol. 54, no. 3, pp. 209226, 1997.

[10] K. Becker, B. Thull, H. Käsmacher-Leidinger et al., "Design and validation of an intelligent patient monitoring and alarm system based on a fuzzy logic process model," Artificial Intelligence in Medicine, vol. 11, no. 1, pp. 33-53, 1997.

[11] A. Singh and J. V. Guttag, "A Comparison of Non-symmetric Entropy-based Classification trees and Support Vector Machine for Cardiovascular Risk Stratification," in Proceedings of the Annual International Conference of the IEEE on Engineering in Medicine and Biology Society (EMBC '11), pp. 79-82, 2011.

[12] D. L. Timms, S. D. Gregory, M. C. Stevens et al., "Hemodynamic modeling of cardiovascular system using mock circulation loops to test cardiovascular devices," in Proceedings of the 33rd Annual International IEEE EMBS Conference, pp. 43014304, Boston, Mass, USA, August-September 2011.
[13] P. Ghorbanian, A. Jalali, A. Ghaffari et al., "An improved procedure for detection of heartarrhythmias with novel preprocessing techniques," Expert Systems. In press.

[14] U. Rajendra Acharya, P. Subbanna Bhat, S. S. Iyengar, A. Rao, and S. Dua, "Classification of heart rate data using artificial neural network and fuzzy equivalence relation," Pattern Recognition, vol. 36, no. 1, pp. 61-68, 2003.

[15] Y. Özbay, R. Ceylan, and B. Karlik, "A fuzzy clustering neural network architecture for classification of ECG arrhythmias," Computers in Biology and Medicine, vol. 36, no. 4, pp. 376-388, 2006.

[16] J. Lacroix and J. Cotting, "Severity of illness and organ dysfunction scoring in children," Pediatric Critical Care Medicine, vol. 6, no. 3, pp. S126-S134, 2005.

[17] A. Jalali, A. Ghaffari, P. Ghorbanian, and C. Nataraj, "Identification of sympathetic and parasympathetic nerves function in cardiovascular regulation using ANFIS approximation," Artificial Intelligence in Medicine, vol. 52, no. 1, pp. 27-32, 2011.

[18] M. Javorka, Z. Lazarova, I. Tonhajzerova et al., "Baroreflex analysis in diabetes mellitus: linear and nonlinear approaches," Medical and Biological Engineering and Computing, vol. 49, no. 3, pp. 279-288, 2011. 

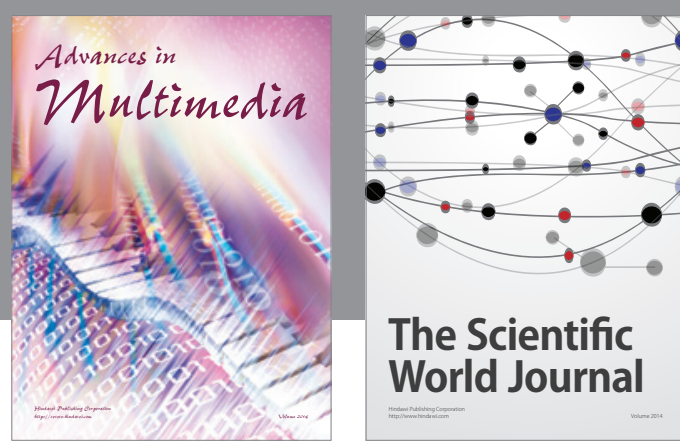

The Scientific World Journal
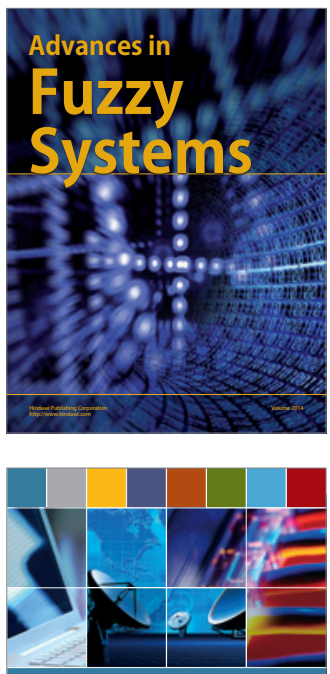

Computer Networks and Communications
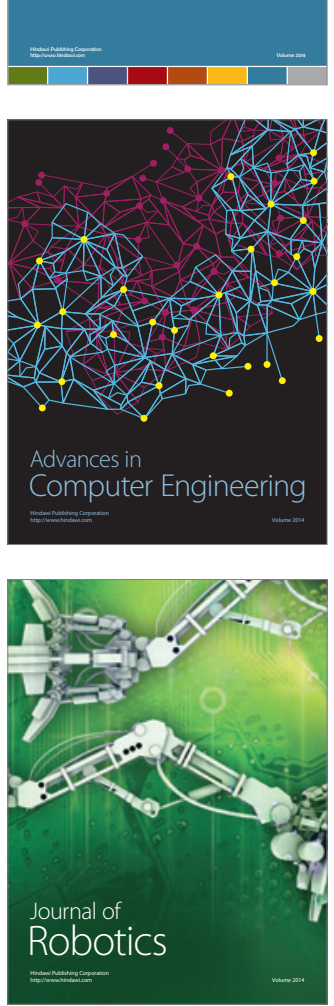
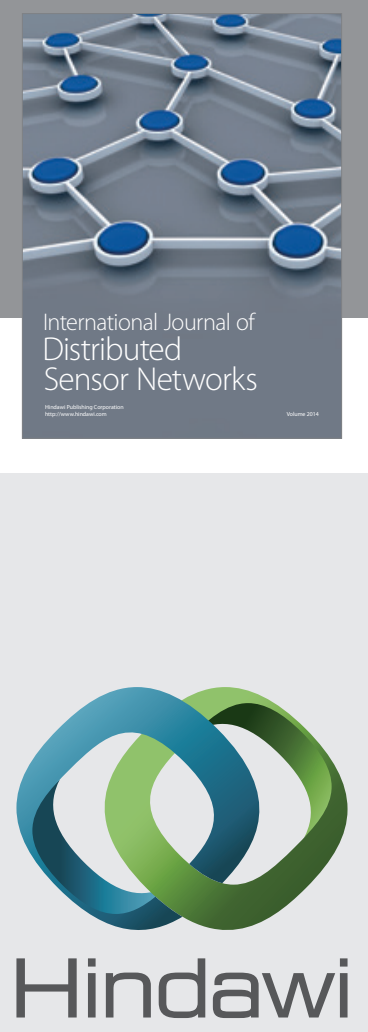

Submit your manuscripts at

http://www.hindawi.com
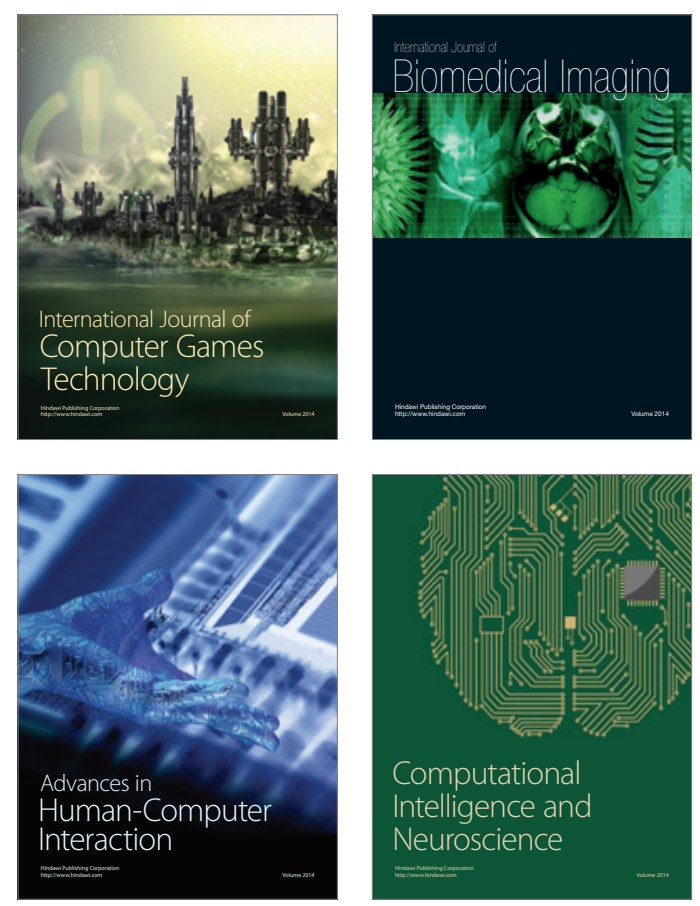
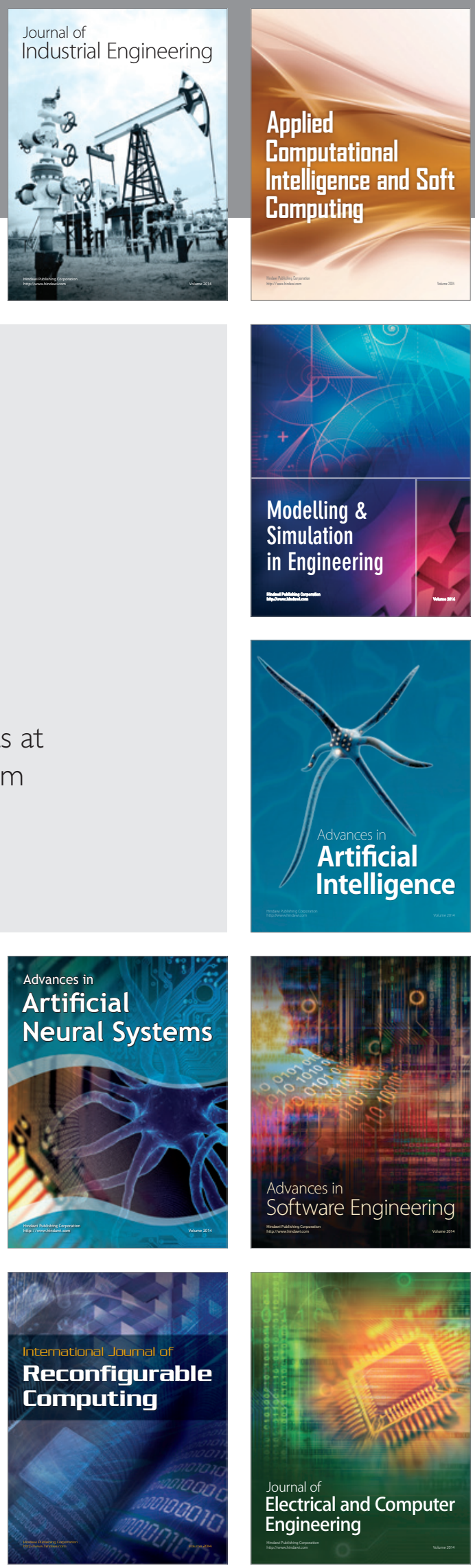